\title{
Results in the development of speech in bilingual children with a cochlear implant
}

\author{
D. Popova, S. Varbanova, Sp. Todorov, Ig. Stancheva \\ Department of ENT, Medical University - Sofia
}

\section{Introduction}

The cochlear implant (CI) is the most important progress in the treatment for children with severe to profound bilateral sensorineural hearing loss who do not receive adequate benefit from hearing aids and making possible better results in auditory, linguistic, social and academic development. Several factors make multilingual proficiency a more attainable goal for CI children than was previously thought. CI offers a profoundly deaf child broader access than do hearing aids to the fine phonetic features and supra segmental patterns of spoken language.

Speech processing replicates the essential components of language to a high degree of resolution that was previously impossible.

CI allows natural language learning, including the overhearing of conversations among native language users.

Such access to incidental language is critical if a child is to learn more than one spoken language via a CI.

Is bilingualism 'special'?

- Bilingualism is the norm in many places.

- More international mobility leads to more bilingual/multilingual families.

- There are many misconceptions about the nature of bilingualism.
- There is a need for information about how bilingualism works.

- The human brain has highest receptivity for language in early infancy and childhood.

- It can acquire more than one language spontaneously without any 'effort'.

\section{Cognitive advantages}

- Bilingual children have higher metalinguistic awareness, i.e. sensitivity to language as a symbolic system. Bilingual children have been found to perform better than monolinguals on some aspects of literacy based on metalinguistic awareness. Bilinguals can 'distance themselves' from language, so to speak, and talk about the form of language separately from its meaning. Many parents also report that bilingual children have more precocious reading skills, and this has recently been confirmed experimentally. Bilinguals seem to have an advantage with respect to some aspects of reading that are related to metalinguistic awareness. A further spin-off of bilingualism is higher awareness of language and greater ability to think about it and talk about it. Bilingual children have a greater ability to focus on the form of language, abstracting away from meaning. Parents of bilingual children often report that their children 
engage in 'language play' that may take the form of 'funny accents' or impossible literal translations between one language and another. CI children from multilingual homes increases, remember that language is a component of the larger issues of culture. Cultures differ widely in their features, including: social customs., communication styles, food and eating rituals, music, roles of parents, grandparents, and extended family, a culture's view of childhood, gender roles, attitudes toward disabilities.

A lot of study supports the belief that exposure to a second language at home does not impair primary language acquisition for some young children with cochlear implants. The study suggests that some children with cochlear implants can learn multiple spoken languages and that parents of such children do not need to avoid using a minority language with their child who has a cochlear implant. The clinical development of the patient correlates to the deprivation and the plasticity of the neural system and is related to the length of deafness, age of implantation and their effect on the performance with a cochlear implant.

The socioeconomic rating, the number of resident members family, higher educational level of family, type and housing conditions and higher occupational level also influence the speech and language development. This case study focuses on the auditory and language development of implanted children - who were exposed in a bilingual environment.

\section{Material and Metods}

All children in this study are assisted at ENT clinic in MU - Sofia and received the CI indication for fitting in the criteria of indication of this center.

This study was conducted with bilateral profound sensorineural hearing loss children (prelingual). All cases had total insertion of electrodes. Both children were hearing aids users prior to the surgery.

\section{Distribution of the two groups by ethnicity}

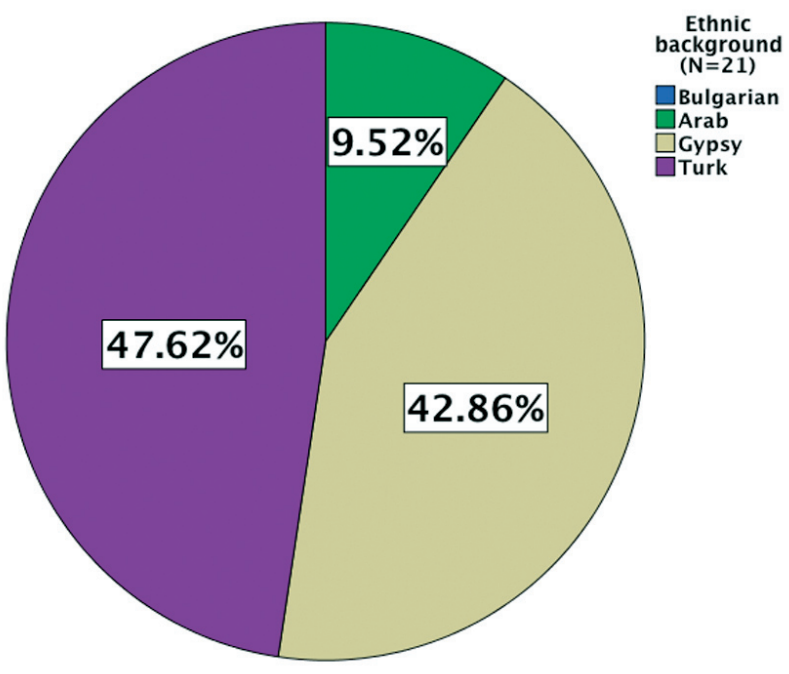

Distribution of the two groups by etyology of hearing loss

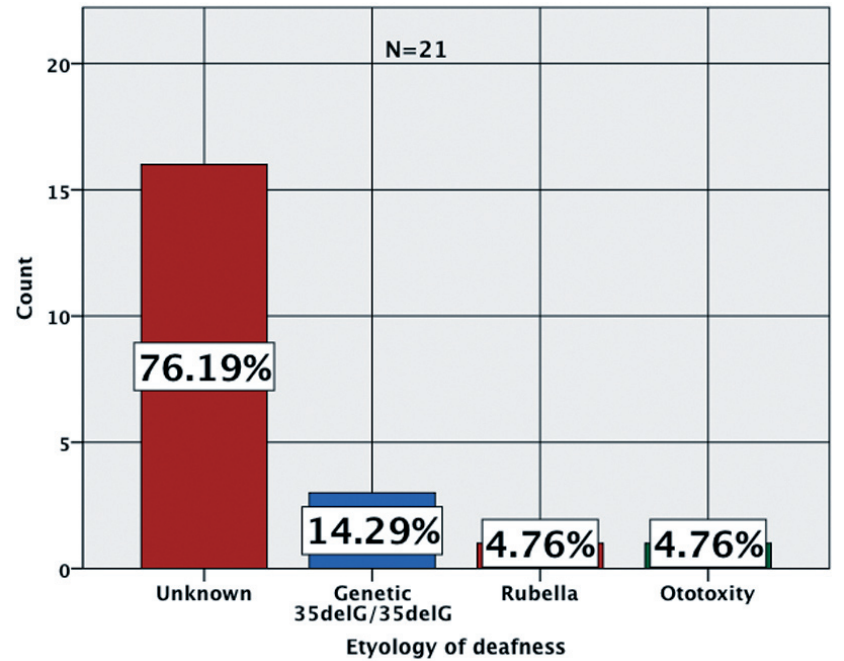

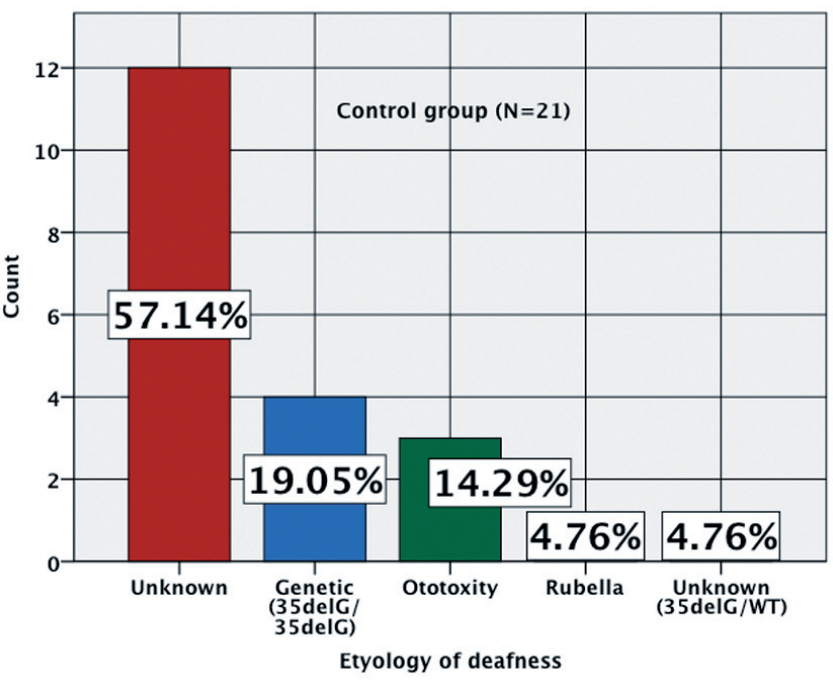


Age of CI in the study group

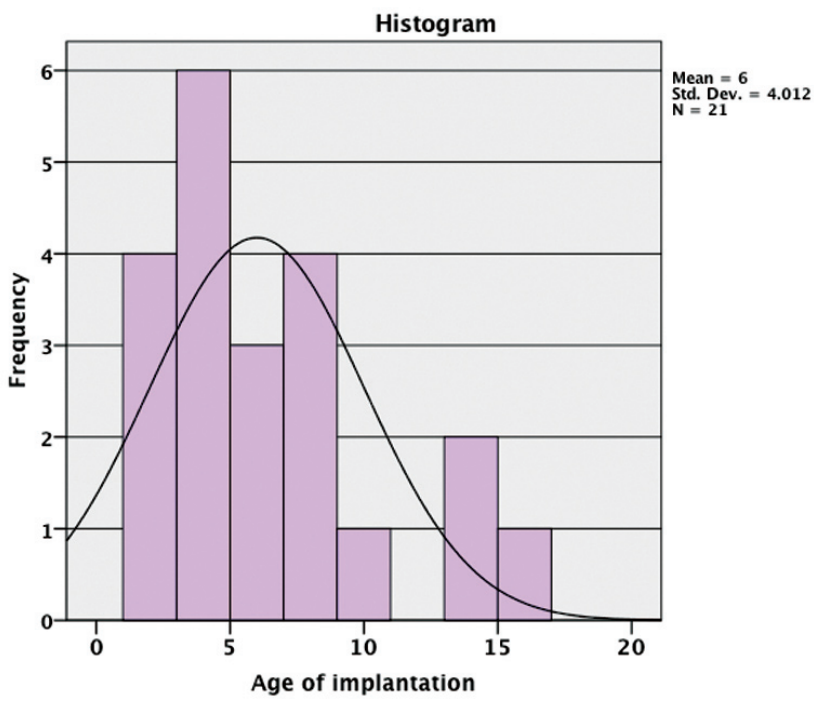

Age of CI in control group

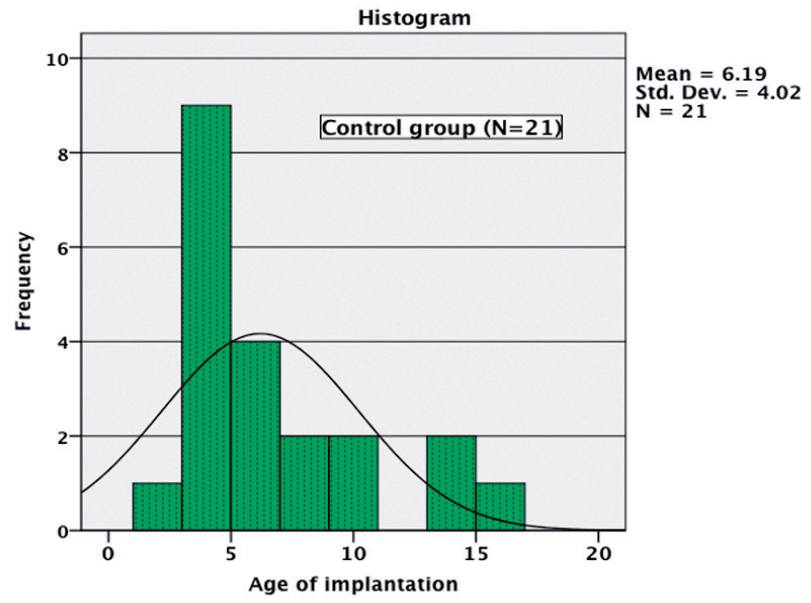

Types of rehabilitation in both groups

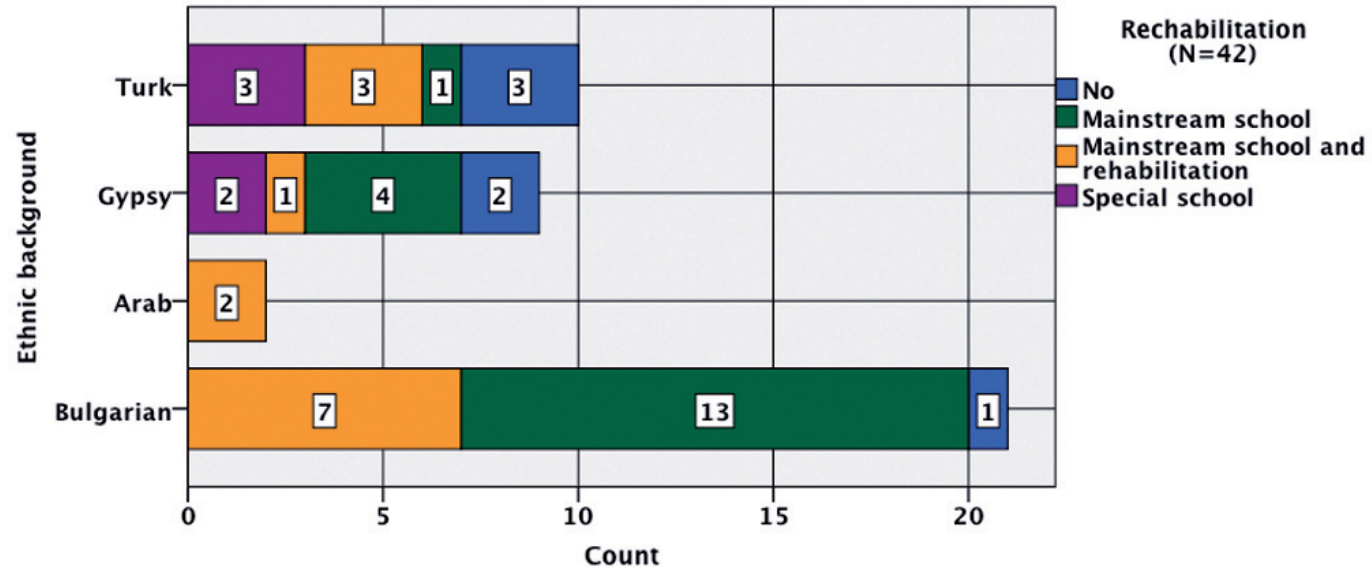

The results are not so good to the official language, naturally all tests are in bulgarian language not in minority language.

\section{Results}

\section{LIP test}

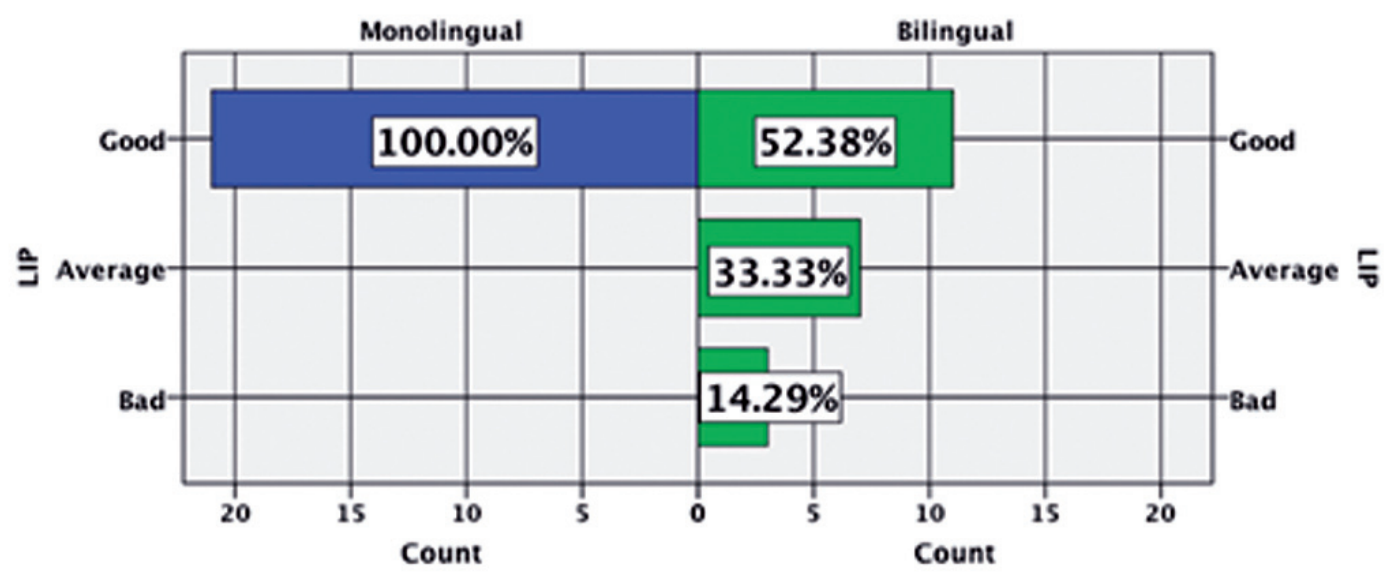


MTP 3 MTP (MonosyllabicTrochee-Polysyllabic Test) Measures the ability to identify different syllable patterns; depending on the age of the child, different word-sets are used (3,6 or 12 items)

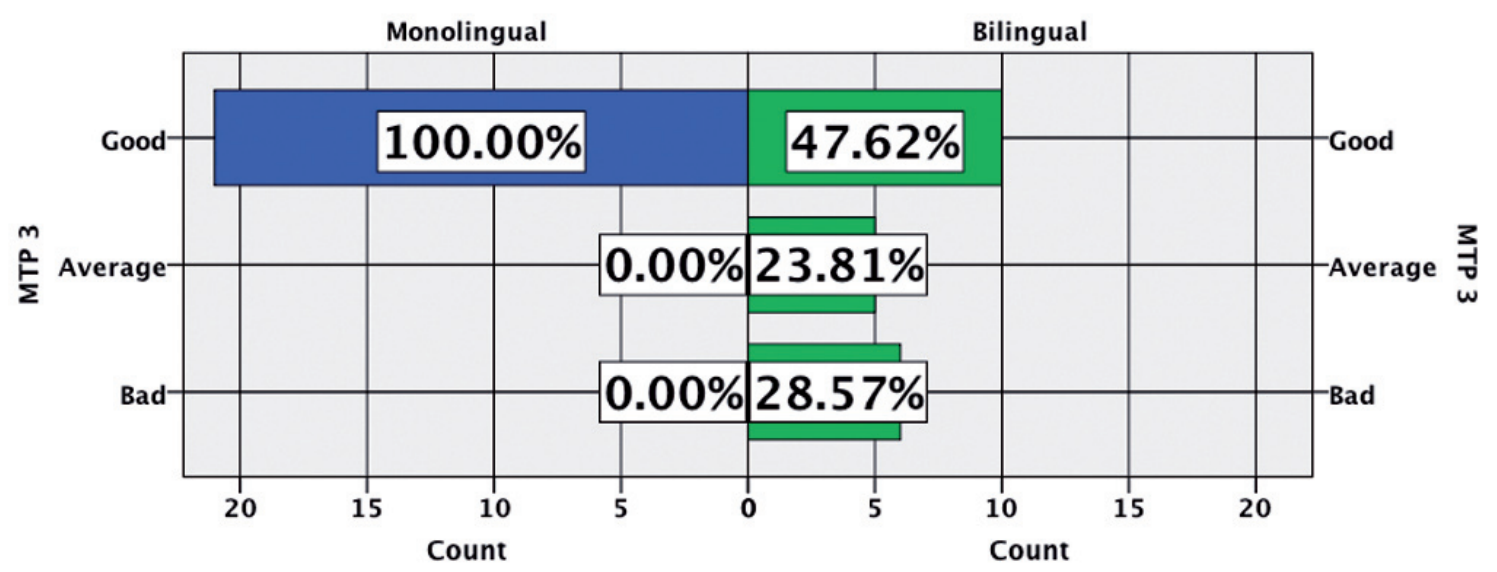

MTP 12 Test

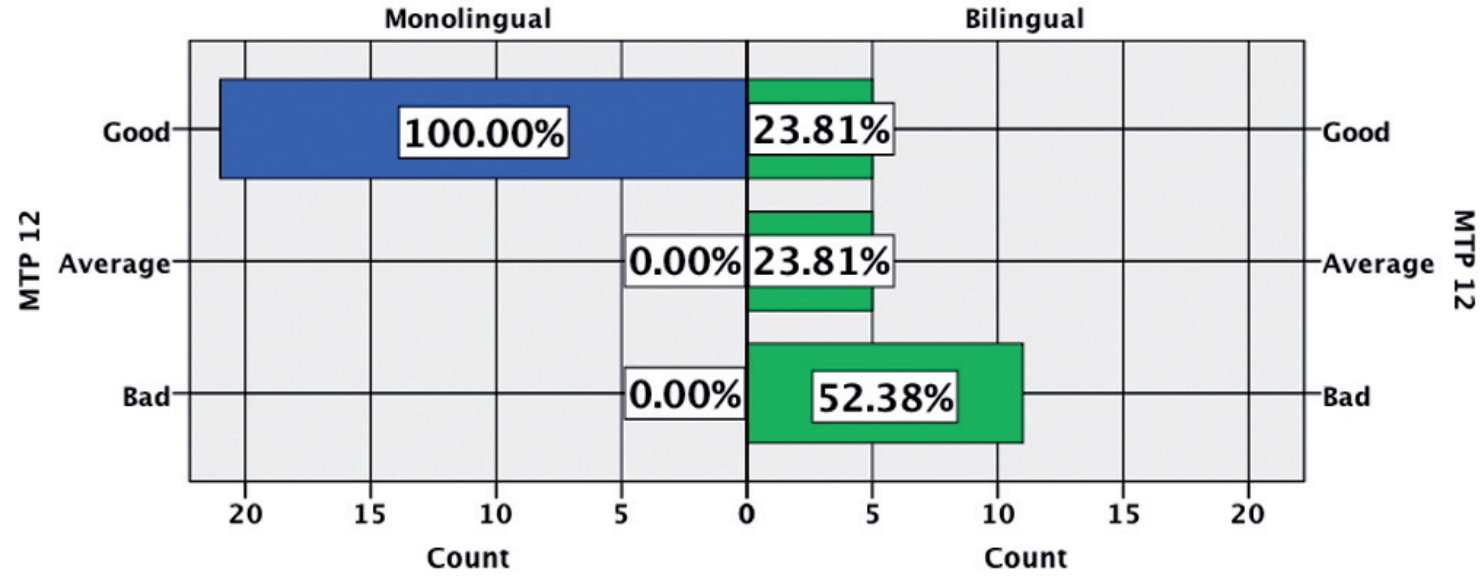

One Sylable Word Test $(p=0,05)$

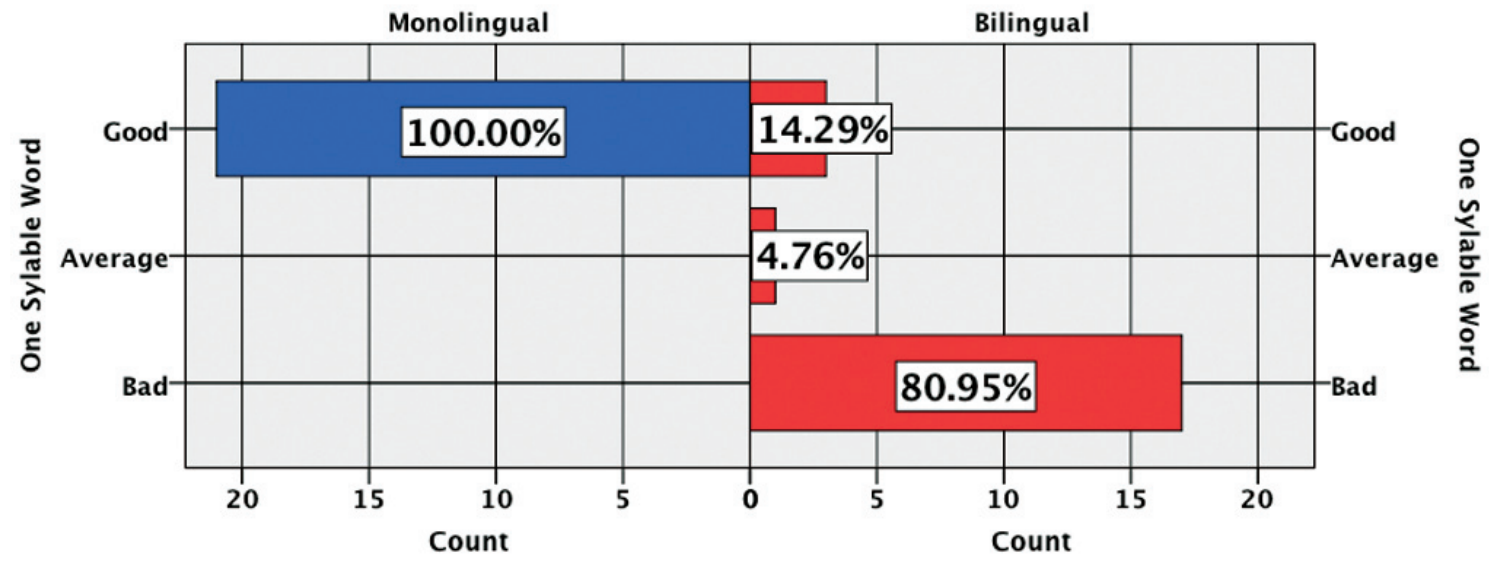


Phoneme $(p=0,05)$

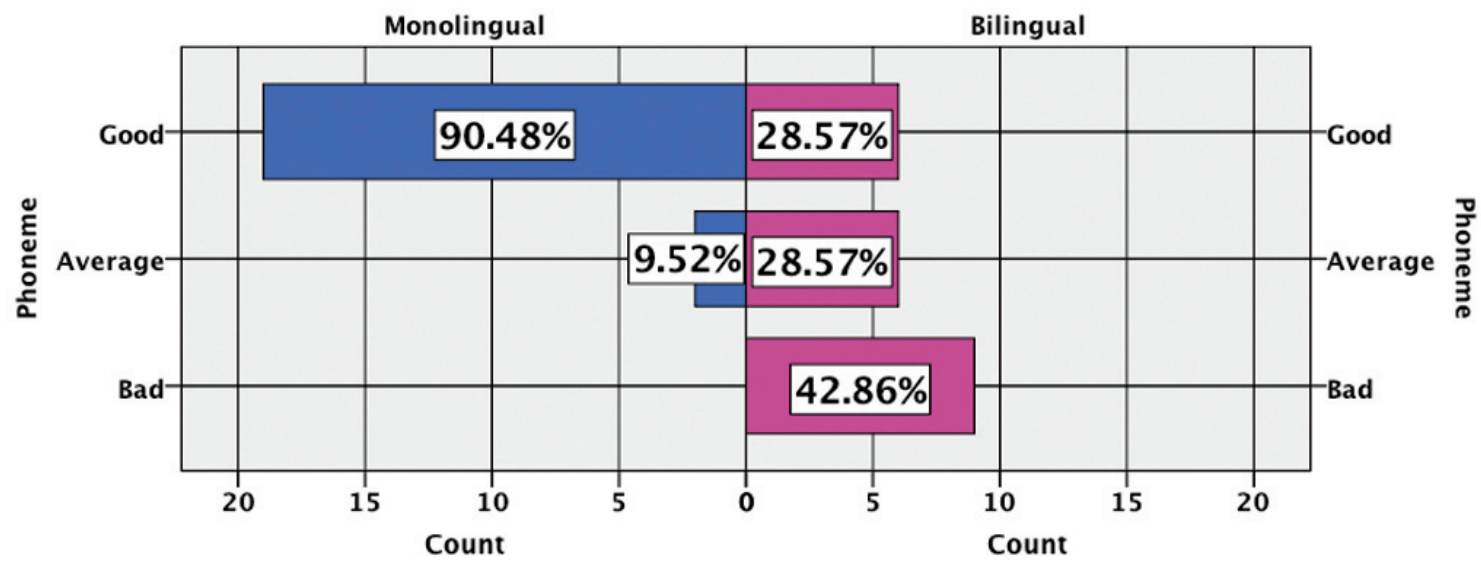

$\operatorname{GASP}(p<0,05)$

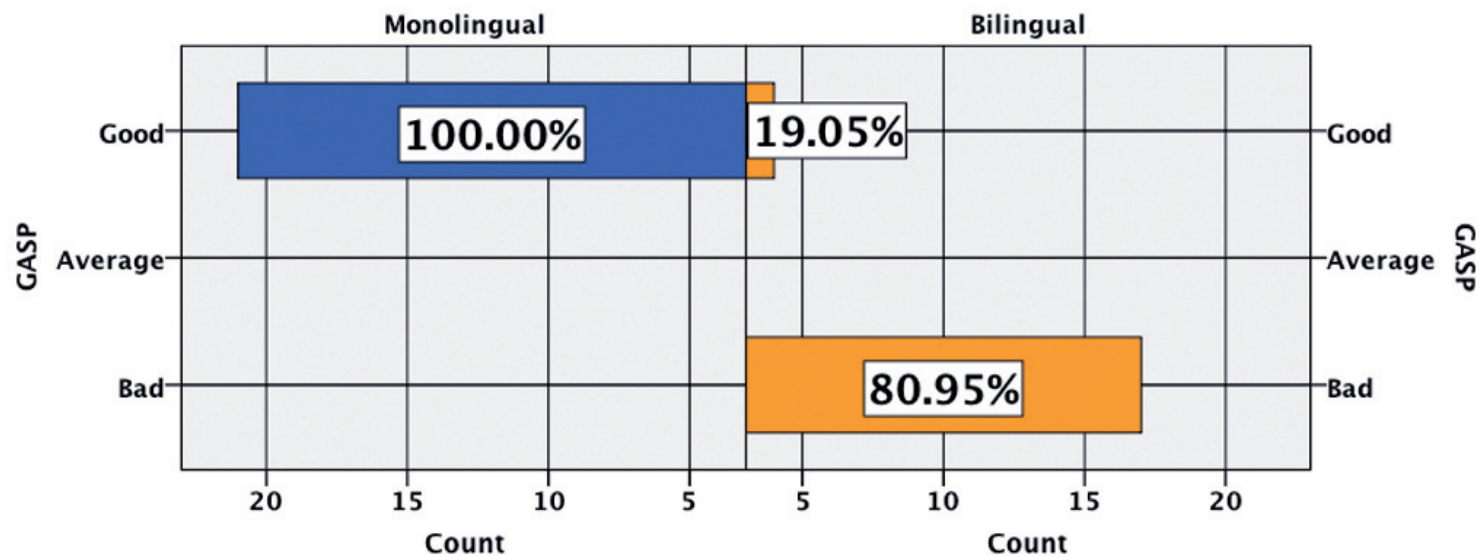

\section{Discussion}

Listening progress Profile (LIP) is a profile to monitor changes in the early auditory performance of young implanted children. The test include the abilities from the response to environmental sounds, discrimination of environmental sounds, discrimination of voice to identification of simple words. The study involved 42 prelingually deaf children, congenitally deaf and implanted before the age of five years. Before the implantation the median score of LIP was zero. By the tree month interval it had increased to 20 , at the 6-month interval to 32 , approximately after 24 months it reached the maximum score available 42 for $52 \%$ of bilingual children. In other tests results are much worse, but they are performed in Bulgarian language. The main reason is that the children are not conducting proper education and the socioeconomic rating of the family is at low level.

\section{In conclusion}

Different tests and there score measure the auditory skills in young implanted children. We have no good results, naturally all tests are in Bulgarian language, not in minority language. Learning two languages from a young age appears to pose many 
problems. If parents hope to raise their children bilingually, it is natural for these children to be exposed to both languages from early in their lives. These children will learn to code-switch between the two languages. They must communicate with their families in one language, but socialize and be educated in another language.

\section{References:}

1. Archbold S., Nikolopoulos T., Tait M., O’Donoghue G., Lutman M. E., Gregory S.(2000). Approach to communication, speech perception and intelligibility after paediatric cochlear implantation. British Journal of Audiology, 34, 257-264.

2. Bhatia T. K., Ritchie W. C. (1999). The bilingual child: Some issues and perspectives. In Ritchie W. C.T. K. Bhatia (Eds.), Handbook of child language acquisition (pp. 569-643). San Diego, CA: Academic Press.

3. Bhatt R., Bolonyai A. (2011). Code-switching and the optimal grammar of bilingual language use. Bilingualism: Language and Cognition, 14, $522-546$.

4. Bouchard M., Ouellet C., Cohen H. (2009). Speech development in prelingually deaf children with cochlear implants. Language \& Linguistic Compass, 3, 1-18. doi:10.1111/j.1749-818X.2008.00079.x 